\title{
Robust power allocation in two-tier heterogeneous networks
}

\author{
Peng Liu, Jiandong Li ${ }^{*}$, Hongyan Li and Kan Wang
}

\begin{abstract}
This paper focuses on a robust distributed power allocation scheme for downlink two-tier heterogeneous networks (HetNets). The objective is to maximize the network aggregate utility of femtocell users (FUEs) which factors the fairness between users under the constraint of not causing serious interference to existing macrocell users (MUEs). Being impractical for different nodes to cooperate in HetNets to obtain precise estimated values of the channel gains between them, it is a challenge to guarantee the performance of the power allocation algorithm by using existing methods. This work makes a step forward in the direction of conquering this challenge by taking into account channel uncertainty, and the robust counterpart of nominal problem, without channel uncertainty, is framed by using worst-case robust optimization theory. To make the robust counterpart computationally tractable, we exploit its convexity and derive that the channel uncertainties between a FUE and nearby femtocell base stations (FBSs) fall into water-filling form being related to the received power from interference sources. Based on the inherent relationship between channel uncertainty and received power, we design a distributed algorithm which merely needs to solve a deterministic problem. The algorithm is devised based on alternating direction method of multipliers (ADMM) with a fast convergence speed. The simulation results demonstrate the robustness of our proposed approach, and the corresponding cost of robustness is investigated.
\end{abstract}

\section{Introduction}

Femtocell base stations (FBSs) are low-power nodes to provide high throughput and customized services to users, and together with macrocells, they form heterogeneous networks (HetNets). Deploying femtocells is an effective and efficient way to increase wireless network capacity, and it provides great benefits to network operators by producing new services. Along with their small size, low cost, and plug-and-play features $[1,2]$, femtocells are considered as a big thing [3] in both existing and future mobile communications. On the other hand, with their marked development, femtocells tend to be largescale and self-organized [4], which brings a big challenge for interference management. More specially, to satisfy femtocell occupants' self-interests, most of them prefer to configure their networks as Closed Subscriber Group (CSG) mode, i.e., users cannot access a specific femtocell without authorization and hence will cause interference

*Correspondence: jdli@mail.xidian.edu.cn

The State Key Lab of ISN \& Information Science Institute, Xidian University, Xi'an, Shaanxi 710071, China to nearby macrocell users (MUEs). Moreover, nearby femtocells would interfere with each other as they become denser, thereby deteriorating network performance.

Some analyses of typical interference scenarios have been made by $[5,6]$. In general, there are two types of interference issues, cross-tier interference and co-tier interference, i.e., interference between a macrocell and femtocells and interference among femtocells. Some stateof-the-art solutions are evaluated in [7], and it indicates that adaptive power control is one of the valid methods to avoid interference regardless of cross-tier and co-tier case. Therefore, herein we emphasize the power allocation of FBSs. The objective is network utility maximization (NUM) with respect to femtocell users (FUEs) under the constraint of not interfering MUEs, which is similar to the underlay scenario in cognitive radio networks.

A considerable literature, such as [8,9], investigated power allocation in HetNets, most of which are under the assumption of perfect channel state information (CSI). We call the corresponding problems nominal, while, unfortunately, the channel uncertainty is normally unavailable in real HetNets due to the lack of complete cooperation

\section{量 Springer}

(C) 2014 Liu et al: licensee Springer. This is an Open Access article distributed under the terms of the Creative Commons Attribution License (http://creativecommons.org/licenses/by/2.0), which permits unrestricted use, distribution, and reproduction in any medium, provided the original work is properly cited. 
between cells. Ignoring channel uncertainty may cause performance loss. For instance, the cross-tier interference as described in Figure 1 shows that to avoid the interference to nearby MUEs, FBS should regulate its power adaptively based on $g_{i, m}$ (channel gain between FBS $i$ and MUE $m$ ). It can be obtained by FBS $i$ from user measurement reports (UMR) of MUE $m$ and the delivery of macrocell base station (MBS) over backhaul. Since backhaul is a delay latency interface, $g_{i, m}$ is generally average, and it is an estimated and imperfect value. An inexact $g_{i, m}$ may lead to the increase of outage probability of MUEs. Moreover, interference between nearby femtocells cannot be ignored in dense scenario. To guarantee the fairness between FUEs, nearby femtocell should consider mutual channel conditions. In the simulation, we illustrate that imperfect CSI may affect the fairness among users.

To mitigate the implication of channel uncertainty, some power control methods model channel gain as a combination of deterministic and uncertain components [10]. In general, there are two approaches to model channel uncertainty, i.e., stochastic approach that assumes statistical knowledge of uncertainty (e.g., a given stochastic distribution) and formulates the problem in a probabilistic manner, and worst-case robust optimization approach where fluctuations of the uncertain component are restricted to be within a bounded and convex set [11]. Due to the stochastic variations in channel gains, the worst-cast approach is more appealing to guarantee MUEs' interference threshold when CSI errors are bounded [12]. Plus the tractability of the worst-case formulation owing to ellipsoid model's neat form, we employ worst-case robust optimization theory to deal with channel uncertainty. More information about the two models can be found in [13] for further study.

Robust optimization theory is first introduced in power control for wireless networks by Yang et al. [11]. Unlike HetNets, they focus on traditional single cellular system. Following [11], several works explore channel uncertainty in cognitive radio networks [10,14-17]. For instance, in [14], Wang et al. devised a robust algorithm to maximize network capacity by joining beamforming and power control for secondary users in cognitive radio networks. In [15], a robust distributed scheme for power control in underlay cognitive radio networks is studied by Parsaeefar et al., where the uncertainties of channel gains between secondary users' base station and primary users are considered. Being similar to the model in [15], we also concentrate on an underlay scenario, i.e., FBSs should not introduce interference to MUEs above a given threshold. At the same time, we consider the uncertainty of channel gains between FBSs and nearby FUEs, which cannot be overlooked in HetNets especially in the densely populated areas. To the best of the authors' knowledge, [18] is the first paper to apply worst-case robust optimization to power control in HetNets. They discuss the effect of channel uncertainty on the Quality of Service (QoS) of MUEs and the energy efficiency of FUEs, whereas we investigate the fairness between FUEs. Moreover, we derive the relationship between the channel uncertainty and received power, which enables the numerical algorithm to merely focus on a deterministic problem.

From a practical perspective, FBSs make decisions individually for their owners' self-interests. Thus, a distributed power allocation scheme is required to meet self-organized feature, which means that FBSs can only

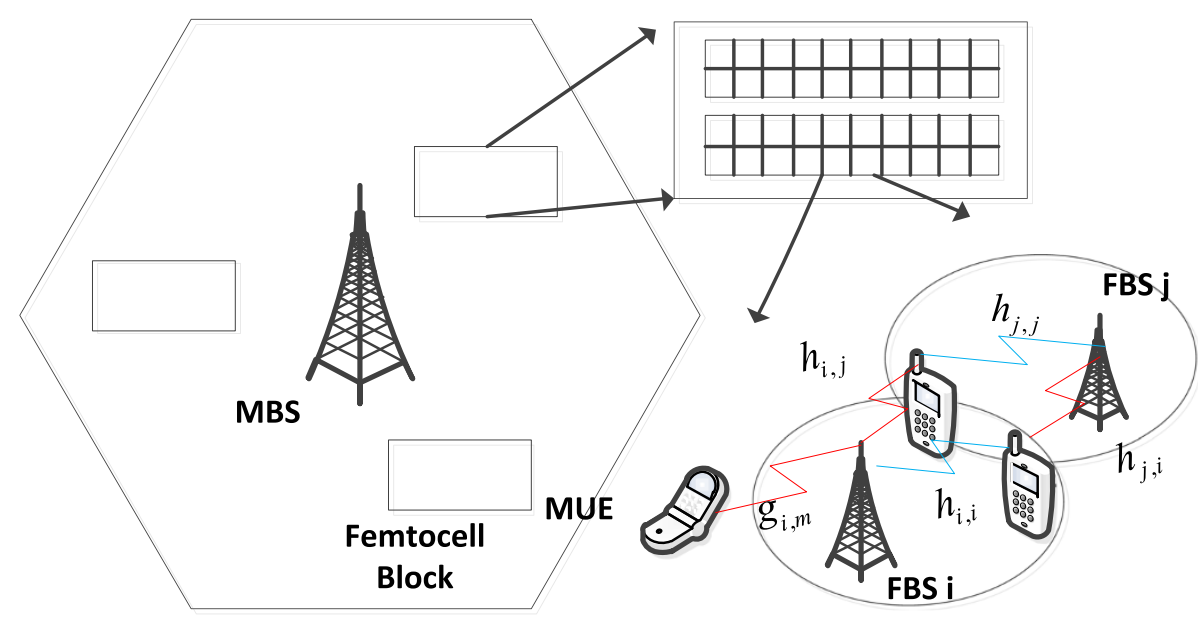

Figure 1 A two-tier heterogeneous wireless network. Consider a two-tier HetNet where a macrocell and femtocells coexisted, and the urban-deployment modeling described [25] is investigated. There exist three femtocell blocks in one macrocell, each of which consists of 40 apartments as illustrated in the top right of this figure. FBS is deployed in each apartment with a certain probability which represents the degree of density. Besides, channel relationship is described in the bottom right of Figure 1. 
exchange information with their neighbors. Some wellknown approaches are dual decomposition (DD) [15,19], alternating direction method of multipliers (ADMM), and game theoretic method. DD decomposes the original problem through Lagrange decomposing method and iterates primal variables and dual variables separately [20]. They are the transmit power of all FBSs, and Lagrange multipliers of the constraints in our scenario. A more promising method is ADMM [21] where each cell determines their users' transmit power and estimates those of other cells, and then reach a consensus by exchanging messages. Compared with DD, the ADMM-based method is known for its fast convergence rate and more robustness against network dynamic and system parameters [22]. Another branch is a series of game theoretic algorithms [23,24]. To use game theoretic method, we first need to exactly define a game. In our scenario, each cell acts as a player in the game, and they alter their actions according to other cells' decisions; in another way, each cell gives a best response to others in the game theoretic paradigm. However, it is normally non-trivial to guarantee the convergence rate and overall performance of power control. Therefore, an ADMM-based algorithm is utilized to implement the power allocation in a distributed manner.

Overall, the contributions of this paper can be summarized as follows:

- To impair the effect of uncertainty of channel gains on the outage probability of MUEs and fairness between FUEs, we devise a NUM problem with the constraint that aggregated interference from FBSs should not affect the communication of MUEs. In this formulation, channel uncertainty is modeled based on worst-case robust optimization theory and $\alpha$-utility function is employed to factor the fairness between FUEs.

- We derive the relationship between received power and channel uncertainty with the help of Karush-Kuhn-Tucker (KKT) systems, which is summarized in Theorem 1. By leveraging Theorem 1, we further exploit the convexity of the robust power allocation problem, making the problem with channel uncertainty to be transformed into a deterministic one in the iterative algorithm.

- The distributed power allocation algorithm is ADMM-based, where each femtocell can perform in a distributed fashion via exchanging messages with neighbors. In the current iteration in ADMM-based method, the robust counterpart with random channel variations can be a deterministic one by filling the channel uncertainty based on UMR in the last iteration. Our proposed algorithm has a fast convergence speed being suitable for the variation of femtocell network environment and robust against the dynamics of network topology.

- We investigate the cost of robustness which means the price we pay for guaranteeing the system to withstand uncertainty. The prices include the decrement of throughput and increment of transmit power investigated in the simulation results.

The rest of this paper is organized as follows. Section 2 introduces the system model and frames power allocation problem without considering channel uncertainty (nominal problem). In section 3, we first present channel uncertainty model and then derive the robust counterpart. The ADMM-based method implemented in a practical system is proposed in Section 4. Section 5 demonstrates the performance of our scheme through numerical simulation, followed by the conclusion in Section 6 .

\section{System model and nominal problem formulation}

Consider a two-tier HetNet where a macrocell and femtocells coexist. To be specific, we investigate the scenario of urban-deployment modeling described in [25], where a macrocell serves $M$ MUEs underlain with $F$ FBSs. $\mathcal{M}=$ $\{1,2, \ldots, M\}$ and $\mathcal{F}=\{1,2, \ldots, F\}$ are the set of MUEs and FBSs, respectively. Without loss of generality, only one user is assumed in each femtocell, indicating that the $i$ th FUE also means the FUE of the $i$ th FBS and the set of FUEs $\left(\mathcal{F}_{u}\right)$ equal to $\mathcal{F}$. Note that our work can be extended to an OFDMA system provided that channels are settled by some channel allocation methods such as those in [26-28]. Compared with suburban-deployment modeling [25], interference between different nodes in this case is more likely to occur, causing a pressing need to design a robust distributed power allocation scheme to mitigate the interference. In this scenario, every femtocell block builds two stripes of apartments, in each of which is deployed a FBS with a predefined probability, deployment ratio [25].

Power allocation is implemented in time slots, which is the minimum scheduling time scale defined in LongTerm Evolution (LTE). MUEs and FUEs are assumed to share the same frequency resource (co-channel deployment). We also assume slow fading channel within a time slot, and channel gains remain constant during that period but may vary in different time slots. Channel gain between the $i$ th FBS and the $m$ th MUE can be expressed as $g_{i, m}$. Analogously, let $h_{i, j}$ be the channel gain between FBS $i$ and FUE $j$.

Let $\gamma_{i}$ be the signal-to-interference-plus-noise ratio (SINR) of the $i$ th FUE which is written as

$$
\gamma_{i}=\frac{p_{i} h_{i, i}}{N_{0}+\sum_{j \neq i} p_{j} h_{j, i}+I_{i, M}}
$$


where $p_{i}$ represents the transmit power of the $i$ th FBS. Interference are from other FBSs, MBS $\left(I_{i, M}\right)$, and thermal noise, which is assumed to be independent and identically distributed (i.i.d) Gaussian distribution with zero mean and $N_{0}$ power. Moreover, let $\mathbf{h}_{i}=\left[h_{1, i}, h_{2, i}, \ldots, h_{F, i}\right]^{T}$ be the channel gain vector for user $i$ containing channel gains from all FBSs. Note that $\mathbf{v}^{T}$ is the transposition operation of vector $\mathbf{v}$.

Recall that FUEs are assumed to communicate in an underlay model, given by

$$
\mathbf{p}^{T} \mathbf{g}_{m} \leq I_{m}^{t}, \quad \forall m \in \mathcal{M},
$$

where $\mathbf{p}=\left[p_{1}, p_{2}, \ldots, p_{F}\right]^{T}$ is a vector consisting of the transmit powers of all $F$ FBSs. $\mathbf{g}_{m}$ is the channel gain vector which is made of channel gains between all FBSs and the $m$ th MUEs, i.e., $\mathbf{g}_{m}=\left[g_{1, m}, g_{2, m}, \ldots, g_{F, m}\right]^{T}$. The underlay scenario requests the interference from FBSs to any MUE $m \in \mathcal{M}$ below an acceptable threshold $I_{m}^{t}$ (interference temperature).

We focus on the following power allocation problem in a two-tier HetNet, where nominal specifies that we do not consider channel uncertainty. Our objective is to find $\mathbf{p}$ to maximize network aggregate utility of all FUEs as well as to avoid interference to any nearby MUE. Therefore, our nominal optimization problem (NoP) can be summarized as

$$
\begin{array}{cl}
\underset{\mathbf{p}}{\operatorname{NoP}: \underset{m a x i m i z e}{\max }} & \sum_{i \in \mathcal{F}_{u}} U_{i}\left(\gamma_{i} / \gamma_{i}^{\mathrm{t}}\right) \\
\text { subject to } & \mathbf{p}^{T} \mathbf{g}_{m} \leq I_{m}^{t}, \quad \forall m \in \mathcal{M}, \\
& 0 \leq p_{i} \leq p^{\max }, \quad \forall i \in \mathcal{F} .
\end{array}
$$

Here, $U_{i}\left(\gamma_{i}\right)=\frac{\gamma_{i}^{1-\alpha}}{1-\alpha}, \alpha>1$, and denote $\gamma_{i}^{\mathrm{t}}$ the SINR target of user $i$. The well-known $\alpha$-utility function is employed to factor fairness between users in that as $\alpha \rightarrow$ $\infty$, the problem converges to a max-min SINR balanced allocation $^{\mathrm{a}}$ [29]. Moreover, another appealing feature is that the $\alpha$-utility function falls in the framework in [19], which makes NoP convex.

\section{Robust counterpart of power allocation}

Due to lack of cooperation and limited feedback between different nodes in two-tier HetNets, channel gains cannot be estimated exactly. In this case, the outage probability of MUEs would increase and the fairness between FUEs would be broken. Thus, channel uncertainty must be taken into account. As defined in robust optimization framework [13], each robust optimization problem consists of three tuples: a nominal formulation which has been presented in NoP, a definition of robustness, and a representation of uncertainty set. We define robustness as the worst-case robustness, namely, the robust counterpart of NoP is always feasible ${ }^{\mathrm{b}}$. For the uncertainty set, a commonly used continuous set surrounding long-term estimate is employed to characterize perturbation errors [11]. The size and shape of the uncertainty set can be devised to characterize the level of perturbations the designer desires to protect against.

Typically, there are three kinds of channel uncertainty models: Polyhedron, D-norm, and Ellipsoid, respectively. They are designed to apply to different application cases [11]. Ellipsoid is generally utilized to model uncertainty caused by channel variations such as those in $[17,30]$. In Ellipsoid model, channel uncertainty set for $\mathbf{g}_{m}$ and $\mathbf{h}_{i}$ can be formulated as

$$
\begin{aligned}
& \mathcal{G}_{m}=\left\{\mathbf{g}_{m}+\Delta \mathbf{g}_{m}: \sum_{i \in \mathcal{F}}\left|\Delta g_{i, m}\right|^{2} \leq \epsilon_{m}^{2}\right\}, \\
& \mathcal{H}_{i}=\left\{\mathbf{h}_{i}+\Delta \mathbf{h}_{i}: \sum_{\mathcal{F}: j \neq i}\left|\Delta h_{j, i}\right|^{2} \leq \zeta_{i}^{2}\right\},
\end{aligned}
$$

where $\Delta \mathbf{g}_{m}=\left[\Delta g_{1, m}, \Delta g_{2, m} \ldots, \Delta g_{F, m}\right]^{T}$ and $\Delta \mathbf{h}_{i}=$ $\left[\Delta h_{1, i}, \Delta h_{2, i}, \ldots, \Delta h_{F, i}\right]^{T} . \epsilon_{m}$ and $\zeta_{i}$ denote the maximal deviation entry of $\mathbf{g}_{m}$ and $\mathbf{h}_{i}$.

Based on the definition of worst-case robustness and the similar expression in $[31,32]$, the robust counterpart of NoP ( $\mathrm{RC}$ for short) by considering the worst-case network utility with respect to channel uncertainty is generally expressed as

$$
\begin{aligned}
\mathrm{RC}: \max _{\mathbf{p}} \min _{\left\{\Delta \mathbf{h}_{i}\right\},\left\{\Delta \mathbf{g}_{m}\right\}} & \sum_{i \in \mathcal{F}_{u}} U_{i}\left(\hat{\gamma}_{i} / \gamma_{i}^{t}\right) \\
\text { subject to } & \mathbf{p}^{T} \hat{\mathbf{g}}_{m} \leq I_{m}^{t}, \forall m \in \mathcal{M} \text {, and (4), } \\
& \hat{\mathbf{g}}_{m} \in \mathcal{G}_{m}, \hat{\mathbf{h}}_{i} \in \mathcal{H}_{i}, \forall m \in \mathcal{M}, \forall i \in \mathcal{F}_{u},
\end{aligned}
$$

where, $\hat{\gamma}_{i}$ has the same expression with $\gamma_{i}$ in (1) except that $\mathbf{h}_{i}$ is replaced by $\hat{\mathbf{h}}_{i}$.

As we can see, the robust counterpart is non-trivial since $\left\{\Delta \mathbf{h}_{i}\right\}$ appears in the denominator of SINR expression. One of the contributions of this paper is that we exploit the convexity of the robust counterpart of NoP and make it trivial. It is effortless to deal with $\left\{\Delta \mathbf{g}_{m}\right\}$ with the help of the conclusion in [13]. Accordingly, (3) in NoP converts into

$$
\mathbf{p}^{T} \mathbf{g}_{m}+\epsilon_{m}\|\mathbf{p}\|_{2} \leq I_{m}^{t}, \quad \forall m \in \mathcal{M},
$$

by adding a protection function [13] in the left side of inequality. However, $\left\{\Delta \mathbf{h}_{i}\right\}$ cannot be handled by directly utilizing the above approach for the reason that $\left\{\Delta \mathbf{h}_{i}\right\}$ is not only in the constraint condition but also in the objective function, and in addition, utility function is nonlinear. To resolve the above issues, by leveraging KKT systems, we derive that the components of $\Delta \mathbf{h}_{i}$ for all $i \in \mathcal{F}_{u}$ follow a neat water-filling form, where the rate of water filling is related to the received power from other cells. Thus, the channel uncertainty can be regarded as the function of 
received power from other cells such that only variables $\mathbf{p}$ are reserved in $\mathrm{RC}$. A compact counterpart of nominal power allocation problem can be obtained, which is summarized in Theorem 1 . For readability, we simply provide the conclusion, and the corresponding proof is elaborated in the Appendix.

Theorem 1. If uncertainty sets for $\mathbf{g}_{m}$ and $\mathbf{h}_{i}$ for all $m \in$ $\mathcal{M}$ and $i \in \mathcal{F}_{u}$ satisfy (5) and (6), respectively, then the robust counterpart of NoP is RoP, expressed as

$$
\begin{aligned}
\text { RoP:maximize } & \sum_{\mathbf{P}} U_{i}\left(\hat{\gamma}_{i} / \gamma_{i}^{t}\right) \\
\text { subject to } & \text { (7) and (4), }
\end{aligned}
$$

where the components of $\Delta \mathbf{h}_{i}$ follow the water-filling form such that

$$
\begin{gathered}
\Delta h_{j, i}=\min \left\{1, \beta^{*} p_{j}\right\} h_{j, i} \\
\sum_{\mathcal{F}: j \neq i}\left(\Delta h_{j, i}\right)^{2}=\zeta_{i}^{2} .
\end{gathered}
$$

and $\beta^{*}>0$ is a constant to relate the channel uncertainty and received power.

Remark. As can be seen in Theorem 1 from (8) and (9), $\left\{p_{j} h_{j, i}\right\}$, the received power of FUE $i$ from FBS $j$, can be interpreted as the rate of water filling and (9) is the termination condition of water filling. That is, we gradually increase $\left\{\Delta h_{j, i}\right\}$ at the rate of the corresponding $\left\{\beta p_{j} h_{j, i}\right\}$, where $\beta$ is the step size of the increase, until either someone's highest level (see (8)) or condition (9) is satisfied. Suppose the number of iterations is $N$, then $\beta^{*}=N \beta$. It implies that if we consider the uncertainty set as an F-1 dimension space, the higher the received power from a FBS is, the more contribution it makes to that direction. In other words, we need to pay more attention to that interfering source, which is consistent with our empirical intuition. This conclusion, to some extent, verifies the practical value of channel uncertainty model.

The major function of Theorem 1 is for designing the iterative method. Since $\left\{\Delta \mathbf{h}_{i}\right\}$ can be expressed by the received power from other FBSs, which can be collected from UMR, the channel uncertainty can be determined in advance in each iteration by using (8) and (9) in Theorem 1. As a result, we simply need to focus on a deterministic problem.

\section{Robust distributed power allocation scheme based on ADMM}

In this section, we leverage the conclusion of Theorem 1 to devise a robust distributed power allocation algorithm which is based on ADMM. In order to do that, we describe some notations as a prerequisite. We define an interference graph $\mathcal{I}=\{\mathcal{V}, \mathcal{E}\}$ with respect to low-tier femtocell networks, which is a directed graph. The vertices $\mathcal{V}$ are FBSs, and we add an edge from $j$ to $i$ provided that there exists an interference $\operatorname{link}^{\mathrm{c}}$ between FBS $j$ and FUE $i$. Let $\mathcal{N}_{i}$ be the set of all the neighbor FBSs that connect with FBS $i$, i.e., the set of user $i$ 's all interfering sources. Since $\left\{\Delta \mathbf{h}_{i}\right\}$ can be expressed by the received power based on Theorem 1 and $\gamma_{i}$ is the function of $\mathbf{p}$ and $\Delta \mathbf{h}_{i}$, informally, we can rewrite $U_{i}\left(\hat{\gamma}_{i} / \gamma_{i}^{t}\right)$ as a function with respect to $\mathbf{p}$ (i.e., $\left.\psi_{i}\left(\mathbf{p}, \Delta \mathbf{h}_{i}(\mathbf{p})\right)\right)$ and hence $\psi_{i}(\mathbf{p})$. Moreover, we introduce local variables $\mathbf{p}^{i}=\left[p_{1}^{i}, \ldots, p_{i}, \ldots, p_{F}^{i}\right]^{T}$ to contain self-transmit power $p_{i}$ for FBS $i$ and its expected transmit power of other cells, $p_{j}^{i}$, for all $j \in \mathcal{F}, j \neq i$. Denoted by $\mathbf{z}_{i, j}$, the consensus variable for any $(i, j) \in \mathcal{E}$ which forces $\mathbf{p}^{i}=\mathbf{p}^{j}$. Finally, we reorganize RoP and reach a ADMM suitable expression, which is called consensus problem (CoP).

$$
\begin{aligned}
\text { CoP: } \underset{\left\{\mathbf{p}^{i}\right\}}{\operatorname{minimize}} & -\sum_{i \in \mathcal{F}_{u}} \psi_{i}\left(\mathbf{p}_{i}\right) \\
\text { subject to } & \mathbf{p}^{i}=\mathbf{z}_{i, j}, \quad \mathbf{p}^{j}=\mathbf{z}_{i, j}, \quad \forall(i, j) \in \mathcal{E}, \\
& \mathbf{z}_{i, j} \in \mathcal{P}, \quad \forall(i, j) \in \mathcal{E} .
\end{aligned}
$$

Here, let $\mathcal{P}=\{\mathbf{p} \mid(7)$ and (4), $\forall m \in \mathcal{M}\}$ be the feasible set of $\mathbf{p}$ which is a closed, nonempty, and convex set. $\chi(\mathbf{p})$ is defined as the indicator function of set $\mathcal{P}$, which means that $\chi(\mathbf{p})=0$ for $\mathbf{p} \in \mathcal{P}$ and $\chi(\mathbf{p})=+\infty$ otherwise. As described in ADMM framework, we define the regularized Lagrangian of $\mathrm{CoP}$ as

$$
\begin{aligned}
\mathcal{L}(\mathbf{p}, \mathbf{z}, \boldsymbol{\lambda}):= & -\sum_{i \in \mathcal{F}_{u}} \psi_{i}\left(\mathbf{p}_{i}\right) \\
& +\sum_{(i, j) \in \mathcal{E}}\left(\lambda_{i, j}^{i T}\left(\mathbf{p}^{i}-\mathbf{z}_{i, j}\right)+\lambda_{i, j}^{j T}\left(\mathbf{p}^{j}-\mathbf{z}_{i, j}\right)\right) \\
& +\sum_{(i, j) \in \mathcal{E}} \frac{1}{\rho}\left(\left\|\mathbf{p}^{i}-\mathbf{z}_{i, j}\right\|_{2}^{2}+\left\|\mathbf{p}^{j}-\mathbf{z}_{i, j}\right\|_{2}^{2}\right),
\end{aligned}
$$

where $\lambda_{i, j}^{i T}$ and $\lambda_{i, j}^{j T}$ denote dual variables or multipliers of constraint (10) in CoP, and $\lambda$ is a vector containing all multipliers. Let $\rho$ be the given cost factor of the $i$ th FBS whose $\mathbf{p}^{i}$ deviates the consensus variable $\mathbf{z}_{i, j}$. Thus, by primaldual decomposition of regularized Lagrangian (12), the ADMM-based recursion in the $(k+1)$-th iteration of CoP can be obtained as 


$$
\begin{aligned}
\mathbf{p}^{i}[k+1]:=\underset{\mathbf{p}^{i}}{\operatorname{argmin}}\left\{-\psi\left(\mathbf{p}^{i}\right)\right. & +\sum_{j \in \mathcal{N}_{i}} \lambda_{i, j}^{i T}[k]\left(\mathbf{p}^{i}-\mathbf{p}^{i}[k]\right) \\
& \left.+\frac{\rho}{2}\left\|\mathbf{p}^{i}-\mathbf{p}^{i}[k]\right\|_{2}^{2}\right\}
\end{aligned}
$$$$
\mathbf{z}_{i, j}[k+1]:=\underset{\mathbf{z}_{i, j} \in \mathcal{P}}{\arg \min }\left\{-\left(\lambda_{i, j}^{i T}[k]+\lambda_{i, j}^{j T}[k]\right) \mathbf{z}_{i, j}\right.
$$$$
\left.+\frac{\rho}{2}\left(\left\|\mathbf{p}^{i}-\mathbf{z}_{i, j}\right\|_{2}^{2}+\left\|\mathbf{p}^{j}-\mathbf{z}_{i, j}\right\|_{2}^{2}\right)\right\}
$$

$\lambda_{i, j}^{i}[k+1]=\lambda_{i, j}^{i}[k]+\rho\left(\mathbf{p}^{i}[k+1]-\mathbf{z}_{i, j}[k+1]\right)$,

$\lambda_{i, j}^{j}[k+1]=\lambda_{i, j}^{j}[k]+\rho\left(\mathbf{p}^{i}[k+1]-\mathbf{z}_{i, j}[k+1]\right)$.

According to the result in [22], (14) can be simplified as

$$
\mathbf{z}_{i, j}[k+1]=\Pi_{p}\left(\frac{1}{2}\left(\mathbf{p}_{i, j}^{i}[k+1]+\mathbf{p}_{i, j}^{j}[k+1]\right)\right),
$$

and dual variables have the relation of $\lambda_{i, j}^{i}[k+1]+\lambda_{i, j}^{j}$ $[k+1]=0$, where $\Pi_{p}$ denotes the projection operation on set $\mathcal{P}$. Through the observation of the above results, FBSs compute local variables $\left\{\mathbf{p}^{i}\right\}$ based on (13) and then send the results to neighbors. Once receiving neighbors' newly updated results, FBSs update their consensus variables $\left\{\mathbf{z}_{i, j}\right\}$ through (17) and then dual variables $\left\{\lambda_{i, j}^{i}\right\}$ according to (15). Finally, all these variables are exchanged between neighbors. We summarize our proposed method in Algorithm 1, where $\sigma$ denotes the predefined error to terminate the algorithm.

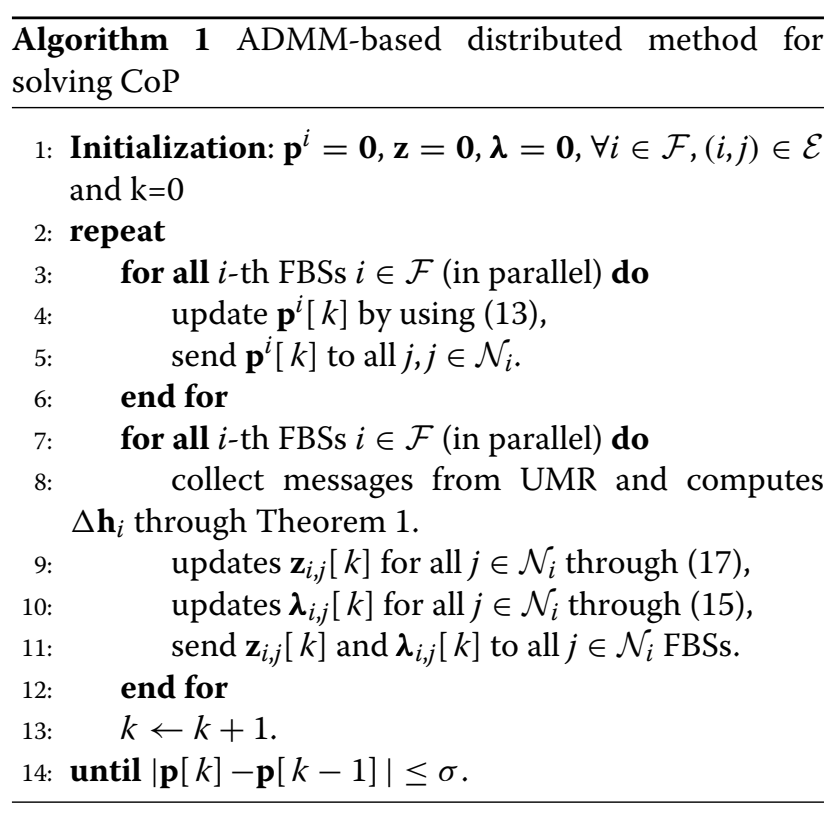

It is noticed that the messages we need to compute $\left\{\Delta \mathbf{h}_{i}\right\}$ are received powers from UMR instead of the ones sent by neighbors.

\section{Numerical simulations}

In this section, we present numerical results to demonstrate the effectiveness of our proposed scheme. The simulation parameters are listed in Table 1 based on [25], and the simulation scenario is illustrated in Figure 1. There exist three femtocell blocks underlain in one macrocell, each of which owns 40 apartments. FBS is deployed in each apartment randomly with probability $d_{r}$ (deployment ratio), ranging from 0.1 to 1 indicating the degree of density. In each femtocell, one user is assumed to be uniformly located. We also assume 40 MUEs randomly dropped in the macrocell. We simulate 2,000 random realizations in terms of MUEs and FUEs to make a statistical average. Gaussian channel model is assumed for simplicity.

\subsection{Evaluation of the channel uncertainty between FBSs and MUEs}

First, the performance of MUEs is evaluated in Figures 2, 3, and 4. Underlay scenario allows for the aggregated interference from FBSs to MUE below a predefined threshold, and thus we take outage probability as performance metric for MUEs. The outage probability is defined as the ratio of the number of MUEs who suffer the interference from FBSs above a predefined threshold $\left(I_{m}^{t}\right)$ to the total number of MUEs. Figure 2 depicts the variation of MUEs' outage probability under different robust parameters with the increase of the number of FBSs $\left(d_{r}\right.$ ranging from 0 to 1$)$. As the number of FBSs grows, aggregate interference causes more MUEs being blocked. Meanwhile, we can see that considering channel uncertainty does bring performance gain in terms of outage probability. The results involving different robust parameters can be illustrated in Figure 3, where we fix $d_{r}=0.8$

Table 1 Simulation parameters

\begin{tabular}{ll}
\hline Parameters & Assumption \\
\hline Carrier frequency & $2,000 \mathrm{MHz}$ \\
Total band & $20 \mathrm{MHz}$ \\
Slot duration & $1 \mathrm{~ms}$ \\
Frame interval & $10 \mathrm{~ms}$ \\
Distance between MBS and FBS & $>35 \mathrm{~m}$ \\
Indoor path loss model & $\mathrm{PL}(\mathrm{dB})=38.46+20 \log _{10} R$ \\
Outdoor path loss model & $\mathrm{PL}(\mathrm{dB})=15.3+37.6 \log _{10} R$ \\
Shadowing standard deviation & $8 \mathrm{~dB}$ \\
Max Tx power FBS & $20 \mathrm{dBm}$ \\
Tx power MBS & $46 \mathrm{dBm}$ \\
\hline
\end{tabular}

$R$ : the distance between the transmitter and receiver. 


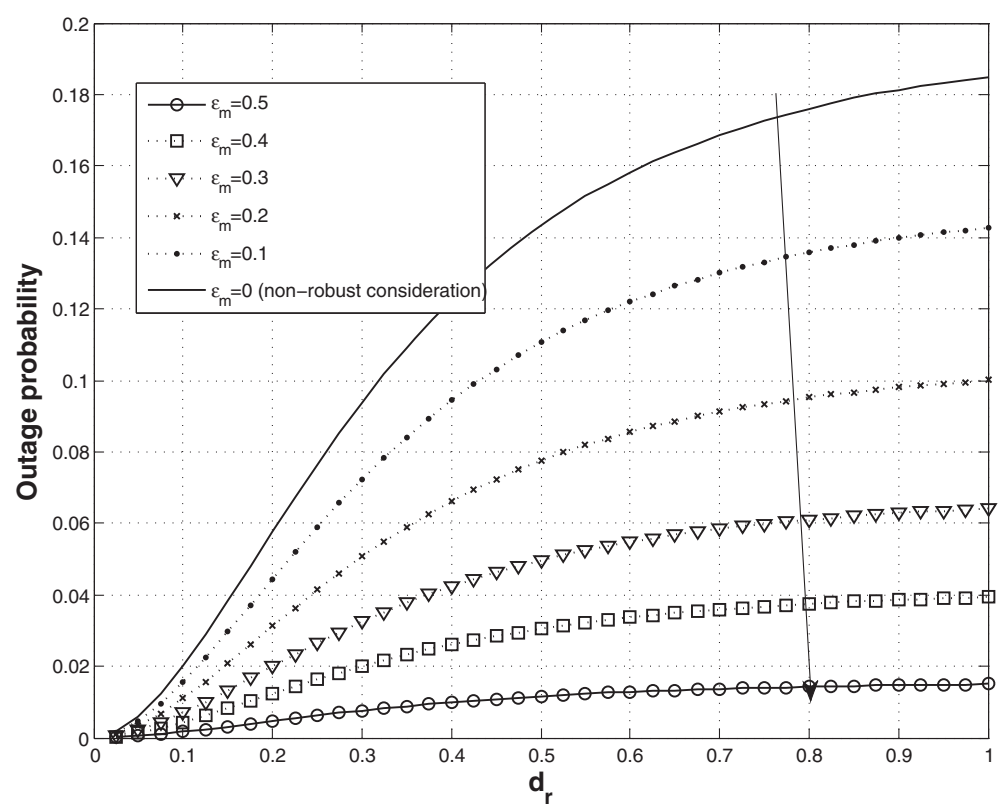

Figure 2 Outage probability vs the density of femtocells. This figure depicts the variation of MUEs' outage probability under different robust parameters with the increase of FBSs, $d_{r}$ ranging from 0 to 1 . As the number of FBSs grows, aggregate interference causes more MUEs being blocked. Meanwhile, we can see that considering channel uncertainty does bring performance gain in terms of outage probability.

to specify the dense case and $I_{m}^{t}=10 N_{0}$. Figure 3 investigates the outage probability in different $\epsilon_{m}$ and compares with non-robust consideration and ideal case which are represented as upper bound and lower bound as baselines. As can be seen, the outage probability decreases steeply initially from $18 \%$ to $1.8 \%$ as $\epsilon_{m}$ increases from 0 to 0.5 . Afterwards, the decreasing rate shows a downward trend till meeting 0 at $\epsilon_{m}=0.7$. This result, to some extent, can be explained by the above analysis, i.e., $\epsilon_{m}$ can be taken as the radius of a ball, and increasing the radius

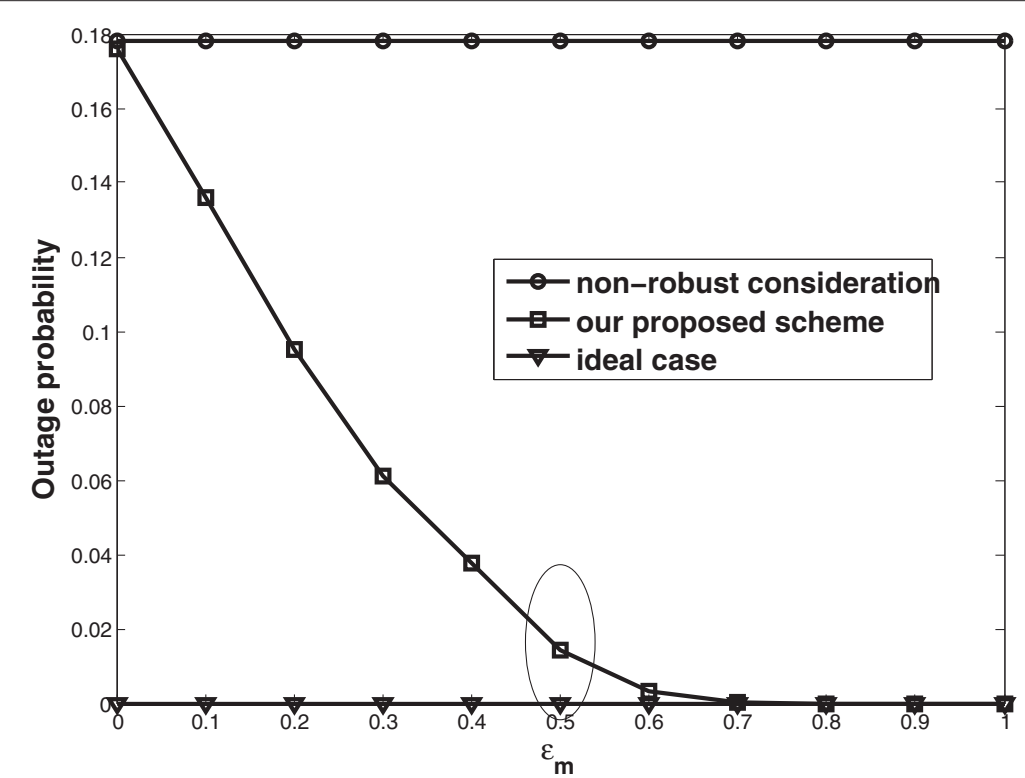

Figure 3 Outage probability vs robust parameter for MUEs. The variation of MUEs' outage probability with the increase of parameter $\epsilon_{m}$ is illustrated, which is compared with worst-case scheme and non-robust scheme when fixing $I_{m}^{t}=10 \mathrm{~N}_{0}$. The worst-case scheme is assumed to totally capture the uncertainty of channel gain where the outage probability is 0 . The outage probability decreases steeply from $18 \%$ to $1.8 \%$ as $\Omega_{m}$ increases from 0 to 0.5. Afterwards, the decreasing rate shows a downward trend till meeting 0 at $\Omega_{m}=0.7$. 


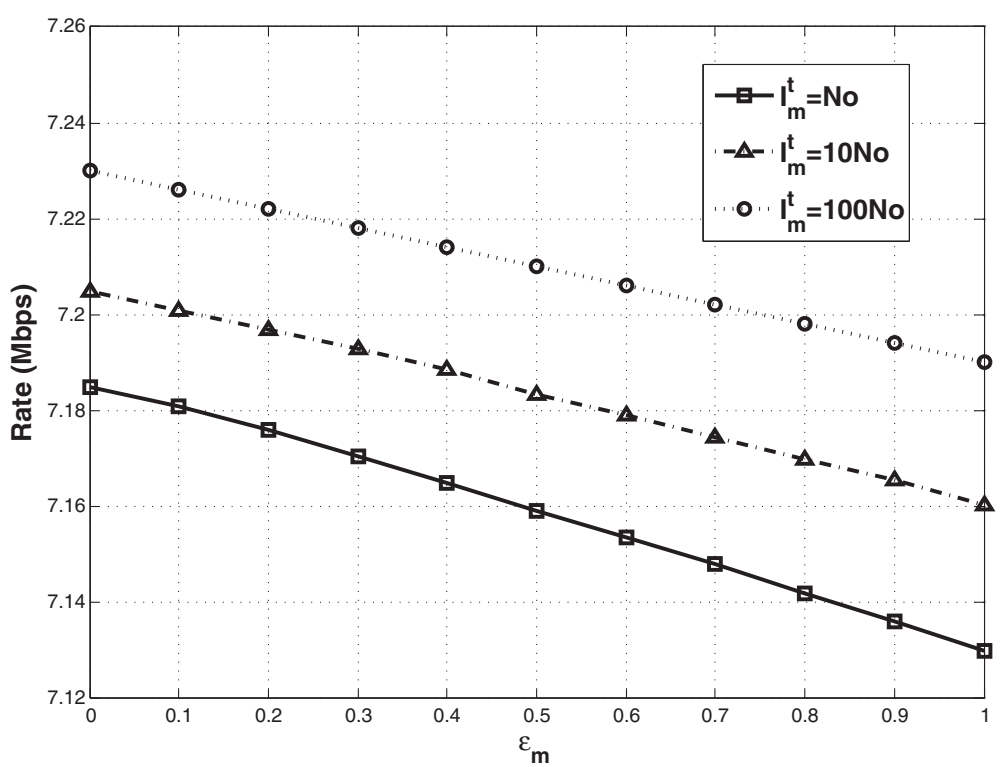

Figure 4 The relationship between $\boldsymbol{\epsilon}_{\boldsymbol{m}}, \boldsymbol{I}_{\boldsymbol{m}}^{\boldsymbol{t}}$, and rates. The average rates of FBSs vary with the increase of $\epsilon_{m}$ from 0 to 1 in different interference thresholds: $I_{m}^{t}=N_{0}, I_{m}^{t}=10 N_{0}$, and $I_{m}^{t}=100 N_{0}$, respectively. Average rates decrease as $\epsilon_{m}$, which is the cost of robustness, increases, and a larger interference threshold tends to obtain higher rates for FBSs.

of a ball will make its volume enlarge steeply. Recall our channel uncertainty model; this corresponds to being capable of containing majority of channel uncertainties by using a moderate value.

Figure 4 investigates the cost of robustness due to considering channel uncertainty between FBSs and MUEs and the relationship between system throughput, robustness parameter $\epsilon_{m}$, and the predefined interference threshold $I_{m}^{t}$. It illustrates the average rates of FBSs with the increase of $\epsilon_{m}$ from 0 to 1 in different interference thresholds: $I_{m}^{t}=N_{0}, I_{m}^{t}=10 N_{0}$, and $I_{m}^{t}=100 N_{0}$, respectively. As we can see, average rates decrease with the increase of $\epsilon_{m}$, and larger interference threshold tends to obtain higher rates for FBSs. To make MUEs immune to the extra interference due to channel uncertainty, we append the protection function to the original optimization model by taking channel uncertainty into account. This will cause FBSs to reduce their transmit power and hence the achievable throughput, which is the cost of robustness. However, by joint observation of Figures 4 and 3 , we can set a proper $\epsilon_{m}$ to attain an acceptable outage probability performance with slight reduction of network rates, e.g., we can specify $\epsilon_{m}=0.5$ in our particular simulation case.

\subsection{Evaluation of channel uncertainty between FBSs and FUEs}

We assess the performance of FUEs on fairness by observing how channel uncertainty affects their SINR distribution, and the relationship between our proposed method and the lower bound which is assumed to obtain exact $\mathrm{CSI}^{\mathrm{d}}$. To capture the worst case, we take the maximum relative difference between the real SINR and SINR target as fairness criteria such as

$$
\max _{i} \frac{\left|\gamma_{i}-\gamma_{i}^{t}\right|}{\gamma_{i}^{t}} \text {. }
$$

Moreover, we define maximum estimated error as $\Delta \mathbf{h}^{\text {max }}$ such that $\mathbb{P}\left\{\Delta \mathbf{h}_{i} \preceq \Delta \mathbf{h}^{\max }\right\} \geq 90 \%$ in the Gaussian channel model.

As can be seen in Figure 5, practical estimated errors have a dramatic effect on worst-case fairness between FUEs without robust consideration (the upper curve). Our proposed robust scheme lies between the curve of nonrobust consideration and the curve of lower bound (which is the result through exact channel estimation) in the simulation. We can see that more fairness can be obtained by increasing robustness, i.e., enlarging the radius of channel uncertainty model.

Meanwhile, in Figure 6, we investigate the cost of robustness like the previous analysis regarding MUEs. Indeed, the extra robustness of fairness against channel uncertainty would not have a big effect on FUEs' rates since we simply remedy the lost fairness caused by channel estimated errors. Note that this does not contradict the trade-off between fairness and throughput, which can be made by regulating fairness parameter $\alpha$ instead of robust parameter $\left\{\zeta_{i}\right\}$. Instead, the cost of robustness in this part is total transmit power consumption of FUEs, as illustrated in Figure 6. It is mainly because that FBSs 


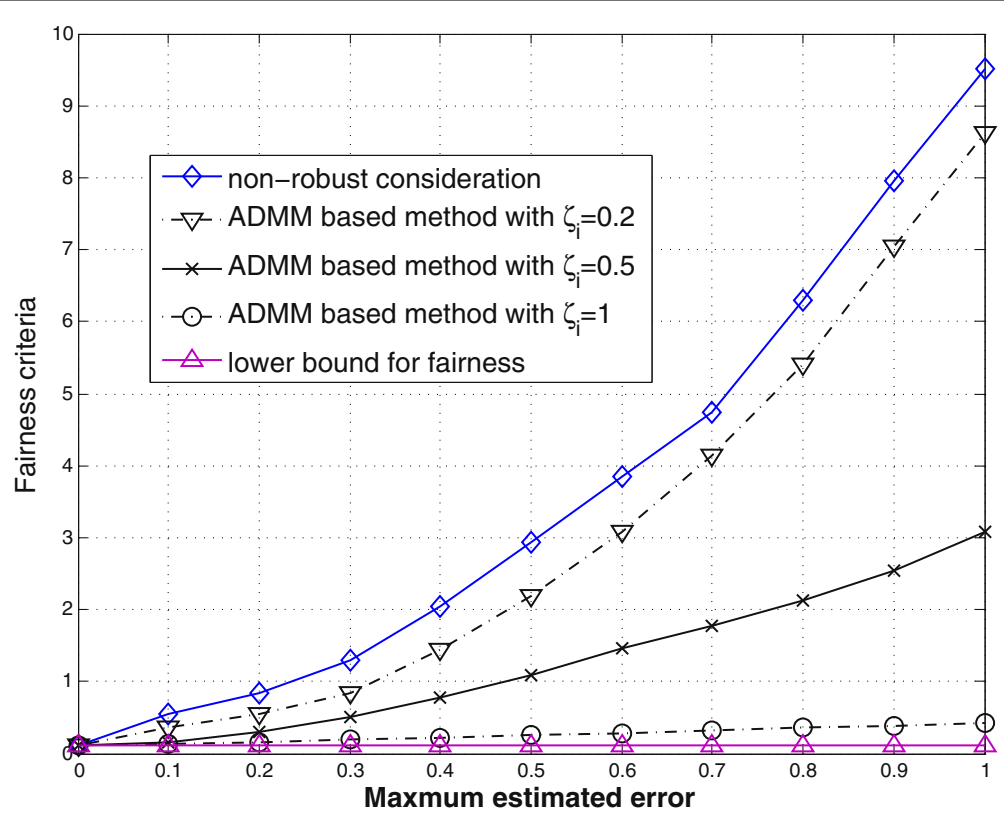

Figure 5 Fairness of FUEs with different schemes. Estimated error $\Delta h_{j, i}$ has a big effect on fairness between FUEs without robust consideration. Lower bound means that exact channel information can be obtained. The ADMM-based distributed method exists between these two curves, and more robust indicates more fairness.

overestimate the interference from other cells and they boost their transmit power compared with non-robust consideration.

\subsection{Evaluation of convergence}

Finally, the convergence of ADMM-based distributed algorithm is shown in Figure 7. The evolution of the transmit power of eight FUEs in the first femtocell block is presented, where $I_{m}^{t}=10 N_{0}, \epsilon_{m}=0.5, \forall m \in \mathcal{M}$, and $\zeta_{i}=0.5, \forall i \in \mathcal{F}_{u}$. From this graph, it can be found that all transmit powers of FBSs converge to the limited values within 10 steps, which verifies its fast convergence rate and hence being suitable for the variations of network environments.

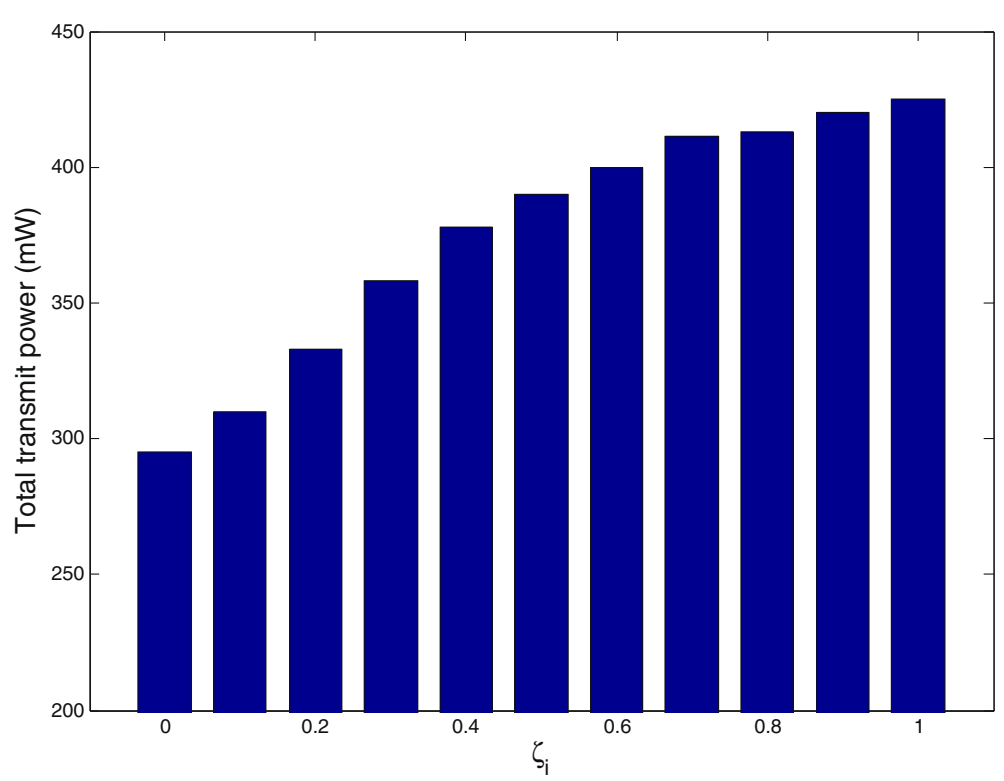

Figure 6 The cost of robustness for protecting fairness. In this figure, maximum estimated error is set to 0.8 . We investigate the variation of power with the increase of robustness, since FBSs overestimate the interference from other cells and they boost their transmission power compared with non-robust consideration. 


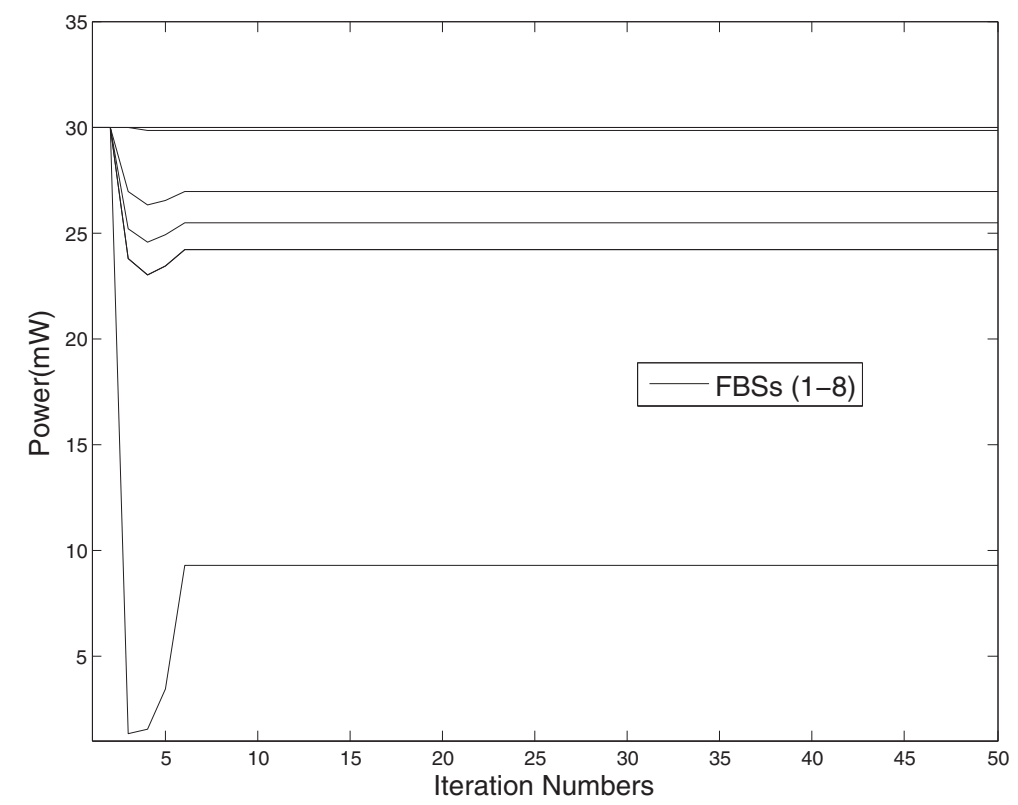

Figure 7 Convergence for the transmit power of FBSs by using ADMM-based distributed method. The evolution of the transmission power of all FUEs in the first femtocell block is presented, where $I_{m}^{t}=10 N_{0}, \epsilon_{m}=0.5$, and $\zeta_{i}=0.5$. All transmit powers of FBSs converge to the limited values within 10 steps, so ADMM-based distributed method converges fast and can adapt to the variation of network situations.

\section{Conclusion}

This paper proposes a robust distributed power allocation scheme in two-tier HetNets, where the uncertainty of channel gains is taken into account. Ellipsoid is used to model the channel uncertainty set for tractability and validity consideration. Under the framework of robust optimization theory, we derive that channel uncertainty can be tackled by using water-filling algorithm, where only received power from neighbor FBSs is involved. This conclusion is very useful for algorithm design, since channel uncertainty can be determined in advance in each iteration. Hence, the closed-form robust counterpart of nominal power allocation is attained. Besides, received power is the rate of water filling, indicating that more power contributes more to channel uncertainty, which accords with our intuition. Moreover, from a practical perspective, we decouple the power allocation problem into a distributed iterative form according to ADMM, which enables our proposed scheme to be suitable for the self-organized feature of femtocells. The ADMM based-algorithm provides fast convergence speed and is robust against system parameters.

The effectiveness of our proposed scheme is demonstrated by simulation results. First, for MUEs, outage probability is taken as performance metric due to underlay deployment of low-tier networks. Our proposed method obtains performance gains at the cost of network achievable throughput since less power is required for interferers to protect MUEs. Second, channel uncertainty has a big impact on the worst-case fairness of FUEs during power allocation in that we can add adequate robust protection for FUEs by enlarging the robust parameter. This overprotection will cost more power of FBSs.

In this paper, only power allocation is taken into account, and how to extend our work with resource scheduling is left for future work. Besides, with the increase of new traffic classes, different traffic classes may need distinct robust criteria. How to allocate resources robustly between users considering different traffic demands also deserves to research.

\section{Appendix 1}

\section{Proof of Theorem 1}

Proof. For simplifying our proving procedure, the interference from macrocells is ignored, and we set $I_{m, i}+$ $N_{0}=1$. Through the observation of problem RC, we can decompose RC into two suboptimization problems, say Sup1 and Sup2. Sup1 is to find $\Delta \mathbf{h}_{i}$ to minimize network utility when transmit power is given, and if $\Delta \mathbf{h}_{i}$ is the function of $\mathbf{p}$ (i.e., $\Delta \mathbf{h}_{i}$ has the form $\Delta \mathbf{h}_{i}(\mathbf{p})$ for certain $\mathbf{p}$ ), Sup2 would become a power allocation problem to maximize network utility with only variable $\mathbf{p}$. In doing this, we first express Sup1 as

$$
\min _{\left\{\Delta \mathbf{h}_{i}\right\}}\left\{\sum_{i \in \mathcal{F}_{u}} U_{i}\left(\hat{\gamma}_{i} / \gamma_{i}^{t}\right), \quad\left\|\Delta \mathbf{h}_{i}\right\|_{2}^{2} \leq \zeta_{i}^{2}, \quad \forall i \in \mathcal{F}_{u}\right\} .
$$


It is noticed that other optimization variables $\left\{\Delta \mathbf{h}_{j} \mid \forall j \neq\right.$ $i, j \in \mathcal{F}_{u}$ \} have no contributions into the objective function $U_{i}\left(\hat{\gamma}_{i} / \gamma_{i}^{t}\right)$ and constraint $\left\|\Delta \mathbf{h}_{i}\right\|_{2}^{2} \leq \zeta_{i}^{2}$. Therefore, Sup1 can be equivalent to the following expression such that $\Delta \mathbf{h}_{i}$ is being optimized independently for all $i$ belonging to $\mathcal{F}_{u}$.

$$
\sum_{i \in \mathcal{F}_{u}}\left\{\min _{\Delta \mathbf{h}_{i}} U_{i}\left(\hat{\gamma}_{i} / \gamma_{i}^{t}\right), \quad\left\|\Delta \mathbf{h}_{i}\right\|_{2}^{2} \leq \zeta_{i}^{2}\right\} .
$$

Since utility function $U_{i}\left(\hat{\gamma}_{i} / \gamma_{i}^{t}\right)$ is a monotonic increasing function with respect to $\hat{\gamma}_{i}$, the objective function of Sup1 can be turned into finding a proper $\Delta \mathbf{h}_{i}$ to minimize $\hat{\gamma}_{i}$, i.e.,

$$
\sum_{i \in \mathcal{F}_{u}}\left\{\min _{\Delta \mathbf{h}_{i}} \hat{\gamma}_{i} / \gamma_{i}^{t},\left\|\Delta \mathbf{h}_{i}\right\|_{2}^{2} \leq \zeta_{i}^{2}\right\} .
$$

For the reason that $\gamma_{i}^{t}$ is a constant and has no effect on the solution when it appears in the objective function, we omit it for brevity. Besides, we introduce an auxiliary variable $t \geq 0$ for the following derivation and finally formulate Sup1 as follows for all $i$ belonging to $\mathcal{F}_{u}$.

$$
\begin{aligned}
& \text { Sup1: } \underset{\Delta \mathbf{h}_{i}, t}{\operatorname{minimize}} t \\
& \text { subject to } \frac{p_{i} h_{i, i}}{1+\sum_{j \neq i} p_{j} \Delta h_{j, i}+\sum_{j \neq i} p_{i} h_{j, i}} \leq t \\
& \left\|\Delta \mathbf{h}_{i}\right\|_{2}^{2} \leq \zeta_{i}^{2} \quad \text { and } \quad t \geq 0 \text {. }
\end{aligned}
$$

To make a compact expression, we set $p_{i} h_{i, i}=\mu_{i}$ and $\sum_{j \neq i} p_{j} h_{j, i}=v_{i}$. For brevity ${ }^{\mathrm{e}},\left\{\Delta h_{j, i}\right\}$ are normalized between 0 and 1 , which can be obtained through dividing by $h_{j, i}$. Accordingly, the constraints of optimization variables themselves are $0 \leq \Delta h_{j, i} \leq 1$ and $t \geq 0$. It is easy to show that Sup1 is a convex optimization problem satisfying the Slater condition [20]. Thus, KKT conditions provide necessary and sufficient conditions for Sup1. We write the complete Lagrange dual function as

$$
\begin{aligned}
\mathcal{L}\left(\lambda_{j, i}, \hat{\lambda}_{j, i}, \lambda_{\zeta}, \lambda_{t}, \lambda_{e}, \Delta \mathbf{h}_{i}, t\right):= & \\
t & -\sum_{j \neq i} \lambda_{j, i} \Delta h_{j, i}+\sum_{j \neq i} \hat{\lambda}_{j, i}\left(\Delta h_{j, i}-1\right) \\
& \lambda_{\zeta}\left(\sum_{j \neq i}\left(\Delta h_{j, i}\right)^{2}-\zeta_{i}^{2}\right)+\lambda_{t} t \\
& \lambda_{e}\left(\mu_{i}-t\left(\sum_{j \neq i} p_{j} \Delta h_{j, i}+v_{i}+1\right)\right),
\end{aligned}
$$

where $\lambda_{j, i}, \hat{\lambda}_{j, i}, \lambda_{\zeta}, \lambda_{t}$, and $\lambda_{e}$ are Lagrange multipliers for the corresponding conditions in Sup1, respectively. The $\mathrm{KKT}$ conditions of this function are provided as

$$
\begin{aligned}
& -t^{*} p_{i} \lambda_{e}^{*}+2 \Delta h_{j, i}^{*} \lambda_{\zeta}^{*}-\lambda_{j, i}^{*}+\hat{\lambda}_{j, i}^{*}=0, \quad \forall j \neq i, \\
& \lambda_{e}^{*}\left(-\sum_{j \neq i} p_{j} \Delta h_{j, i}^{*}-v_{i}-1\right)+1-\lambda_{t}^{*}=0, \\
& \lambda_{j, i}^{*}\left(-\Delta h_{j, i}^{*}\right)=0, \\
& \lambda_{t}^{*} t^{*}=0, \\
& \hat{\lambda}_{j, i}^{*}\left(\Delta h_{j, i}^{*}-1\right)=0 .
\end{aligned}
$$

Here, the variables with superscript $*$ are the optimal values of the primary variables and dual variables. Note that $t>0$, we obtain $\lambda_{t}^{*}=0$ from (21d). Then (21a) can be rewritten as

$$
\lambda_{j, i}^{*}=-t^{*} p_{j} \lambda_{e}^{*}+2 \Delta h_{j, i}^{*}+\hat{\lambda}_{j, i}^{*}
$$

Substituting (22) into (21c), we derive

$$
\left(-t^{*} p_{j} \lambda_{e}^{*}+2 \Delta h_{j, i}^{*}+\hat{\lambda}_{j, i}^{*}\right) \Delta h_{j, i}^{*}=0 .
$$

Suppose $\lambda_{j, i}^{*}>0$, then it is apparent that $\Delta h_{j, i}^{*}=0$ (from (21c)), and hence, according to (21e), $\hat{\lambda}_{j, i}^{*}$ will be greater than 0 . Thus, from (22), $-t^{*} p_{j} \lambda_{e}^{*}>0$ must hold. Such a conclusion is contradictory to the given conditions, that is, all three terms on the left side of inequality are nonnegative. For this reason, $\lambda_{j, i}^{*}=0$ should be satisfied, wherein $-t^{*} p_{j} \lambda_{e}^{*}+2 \Delta h_{j, i}^{*}+\hat{\lambda}_{j, i}^{*}=0$. Plus the condition of (21e), we obtain that $\Delta h_{j, i}^{*}$ should not be equal to zero if only $p_{j}>0$, which indicates that if the $j$ th FBS induces interference to the $i$ th FUEs, the corresponding channel uncertainty must be taken into account. Therefore, we derive $\Delta h_{j, i}^{*}=\left(t^{*} p_{j} \lambda_{e}^{*}-\lambda_{j, i}^{*}\right) / 2 \lambda_{\zeta}^{*}$.

Similarly, we rewrite (21a) as $\hat{\lambda}_{j, i}^{*}=t^{*} p_{j} \lambda_{e}^{*}-2 \Delta h_{j, i}^{*}+\lambda_{j, i}^{*}$ and then make an analysis of KKT conditions analogous to the above methods. Recall that $\left\{\Delta h_{j, i}^{*}\right\}$ are normalized at the beginning of the proof; we restore the results by multiplying by $\left\{h_{j, i}\right\}$. Finally, we achieve a significant conclusion, which is written in a closed form.

$$
\begin{gathered}
\Delta h_{j, i}^{*}=\min \left\{1, \beta^{*} p_{j}\right\} h_{j, i}, \\
\sum_{\mathcal{F}_{u}: j \neq i}\left(\Delta h_{j, i}^{*}\right)^{2}=\zeta_{i}^{2},
\end{gathered}
$$

where $\beta^{*}=t^{*} \lambda_{e}^{*} / 2 \lambda_{\zeta}^{*}$. By observing the above expressions, it is a water-filling form, where $\left\{p_{j} h_{j, i}\right\}$ (the received power from FBS $j$ to FUE $i$ ) can be regarded as the rate of water filling and (25) represents the terminal condition of water filling. Thus, we finish the proof of Theorem 1.

\section{Endnotes}

${ }^{a}$ In SINR balanced allocation, the objective function is $\operatorname{maxmin}_{\mathbf{p}} \frac{\gamma_{i}}{\gamma_{i}^{\mathrm{t}}}$. 
${ }^{\mathrm{b}}$ This definition does not mean that we can capture all channel variations in practice. Indeed, it can be obtained through expanding the uncertain set, but we will not intend to do this. The purpose of the paper is to instruct the designer to select a proper uncertainty set in order to gain a considerable robustness at only a small cost of performance. Therefore, this definition is only applicable to the problem formulation rather than real situation.

${ }^{\mathrm{c}}$ Informally, if the interference from FBS $j$ to FUEs $i$ is below a given threshold, we say, there exists an interference link between FBS $j$ to FUE $i$.

${ }^{\mathrm{d}}$ Since we do not set $\alpha$ in $\alpha$-utility to infinity, it is impossible for all FUEs to meet their SINR requirement. Thus, there exists a lower bound which is greater than 0 .

${ }^{\mathrm{e}}$ Note that, $\left\{\Delta h_{j, i}\right\}$ cannot be negative for worst-case consideration since they appear in the denominator of SINR expression.

\section{Abbreviations}

ADMM: Alternating direction method of multipliers; CoP: Consensus problem: CSG: Closed Subscriber Group; CSI: Channel state information; DD: Dual decomposition method; FBS: Femtocell base station; FUEs: Femtocell users: HetNet: heterogeneous network; KKT: Karush-Kuhn-Tucker; LTE: Long-Term Evolution; MBS: Macrocell base station; MUEs: macrocell users; NoP: Norma power allocation problem; NUM: Network utility maximization; RC: Robust counterpart of NoP in general expression; RoP: Robust counterpart of NoP; SINR: Signal-to-interference-plus-noise ratio; Sup1: $1_{\text {st }}$ suboptimization problem of RC; Sup2: 2nd suboptimization problem of RC; UMR: User measurement report.

\section{Competing interests}

The authors declare that they have no competing interests.

\section{Acknowledgements}

This work was supported by the National Natural Science Foundation of China under Grants No. 60972047 and No. 61231008; the National Science and Technology Major Project under Grants No. 2011ZX03005-004, No. 2011ZX03004-003, No. 2011ZX03005-003-03, and No. 2013ZX03004007-003; the Shannxi 13115 Project under Grant No. 2010ZDKG-26; the National Basic Research Program of China (973 Program) under Grant No. 2009CB320404; the Program for Changjiang Scholars and Innovative Research Team in University under Grant No. IRT0852; the 111 Project under Grant No. B08038; and the State Key Laboratory Foundation under Grants No. ISN1002005, and No. ISN090305.

Received: 26 June 2013 Accepted: 14 April 2014

Published: 28 April 2014

\section{References}

1. V Chandrasekhar, J Andrews, A Gatherer, Femtocell networks: a survey Commun. Mag. IEEE. 46(9), 59-67 (2008)

2. J Boccuzzi, M Ruggiero, Femtocells:Design \& Application. (McGraw-Hill Professional, New York, 2010)

3. Small Cell, the next BIG thing-The business case for residential small cells as part of a high leverage network. [http://www.alcatel-lucent.com/wps/ DocumentStreamerServlet?LMSG_CABINET=Docs_and_Resource_Ctr \&LMSG_CONTENT_FILE=Other/May2011_Small_Cells_EN_BusCase Overview.pdf\&UNIQUE_NAME=\&lu_lang_code=en_WW]. Accessed 20 Jan 2013

4. JG Andrews, H Claussen, M Dohler, S Rangan, MC Reed, Femtocells: past, present, and future. Selected Areas Commun. IEEE J. 30(3), 497-508 (2012)

5. D Knisely, T Yoshizawa, F Favichia, Standardization of femtocells in 3GPP. Commun. Mag. IEEE. 47(9), 68-75 (2009)

6. M Yavuz, F Meshkati, S Nanda, A Pokhariyal, N Johnson, B Raghothaman, A Richardson, Interference management and performance analysis of UMTS/HSPA+ femtocells. Commun. Mag. IEEE. 47(9), 102-109 (2009)
7. N Saquib, E Hossain, LB Le, DI Kim, Interference management in OFDMA femtocell networks: issues and approaches. Wireless Commun IEEE. 19(3), 86-95 (2012)

8. V Chandrasekhar, JG Andrews, T Muharemovic, Z Shen, A Gatherer, Power control in two-tier femtocell networks. Wireless Commun. IEEE Trans. 8(8), 4316-4328 (2009)

9. DT Ngo, LB Le, Ngoc Le-T, E Hossain, DI Kim, Distributed interference management in two-tier CDMA femtocell networks. Wireless Commun. IEEE Trans. 11(3), 979-989 (2012)

10. S Gong, P Wang, Y Liu, W Zhuang, Robust power control with distribution uncertainty in cognitive radio networks. Selected Areas Commun. IEEE J. 31(11), 2397-2408 (2013)

11. K Yang, Y Wu, J Huang, X Wang, Verdú S, Distributed robust optimization for communication networks, in Proceedings of IEEE the 27th Conference on Computer Communications (INFORMCOM 2008) (Phoenix, AZ, USA, 15-17 April 2008, pp. 1157-1165

12. S Parsaeefard, AR Sharafat, Robust worst-case interference control in underlay cognitive radio networks. Vehicular Technol. IEEE Trans. 61(8), 3731-3745 (2012)

13. A Ben-Tal, L El Ghaoui, A Nemirovski, Robust Optimization. Princeton University Press, Princeton and Oxford, 2009)

14. F Wang, W Wang, Robust beamforming and power control for multiuser cognitive radio network, in Proceedings of IEEE Global Telecommunication Conference (GLOBECOM 2010) (Miami, Florida, USA, 6-10 December 2010, pp. 1-5

15. S Parsaeefard, A Sharafat, Robust distributed power control in cognitive radio networks. Mobile Comput. IEEE Trans. 12(4), 609-620 (2013)

16. S Sun, W Ni, Y Zhu, Robust power control in cognitive radio networks: a distributed way, in Proceedings of 2011 IEEE International Conference on Communications (ICC) Kyoto, Japan, 5-9 June 2011, pp. 1-6

17. UL Wijewardhana, M Codreanu, M Latva-aho, Robust beamformer design for underlay cognitive radio network using worst case optimization, in Proceedings of the 11th International Symposium on Modeling Optimization in Mobile, Ad Hoc \& Wireless Networks (WiOpt) Tsukuba Japan, 13-17 May 2013, pp. 404-411

18. X Guan, Q Han, K Ma, X Wang, Robust uplink power control for co-channel two-tier femtocell networks. AEU-Int. J. Electron. Commun. 67(6), 504-512 (2013)

19. S Stanczak, M Wiczanowski, H Boche, Fundamentals of Resource Allocation in Wireless Networks: Theory and Algorithms, vol. 3. Springer-Verlag, Berlin Heidelberg, 2009)

20. S Boyd, L Vandenberghe, Convex Optimization. (Cambridge University Press, New York, 2004)

21. S Boyd, N Parikh, E Chu, B Peleato, J Eckstein, Distributed optimization and statistical learning via the alternating direction method of multipliers. Foundations Trends ${ }^{\circledast}$, Mach Learn. 3, 1-122 (2011)

22. A Simonetto, G Leus, On distributed optimization, convex relaxations, and sensor network localization problems. arXiv preprint arXiv:1309.2502 (2013)

23. D Gesbert, SG Kiani, A Gjendemsj, GE Oien, Adaptation, coordination, and distributed resource allocation in interference-limited wireless networks. Proc. IEEE. 95(12), 2393-2409 (2007)

24. D Schmidt, C Shi, R Berry, M Honig, W Utschick, Distributed resource allocation schemes. Signal Process. Mag. IEEE. 26(5), 53-63 (2009)

25. 3GPP, Simulation assumptions and parameters for FDD HeNB RF requirements, in TSG RAN WG4 Meeting 51, R4-092042 (Alcatel-Lucent, picoChip Designs and Vodafone, 2009)

26. R Knopp, PA Humblet, Multiple-accessing over frequency-selective fading channels, in Proceedings of Sixth IEEE International Symposium on Personal, Indoor and Mobile Radio Communications (PIMRC'95), vol. 3 (Toronto, Ontario, Canada, 27-29 Sept 1995, pp. 1326-1330

27. I Toufik, R Knopp, Channel allocation algorithms for multi-carrier systems, in Proceedings of IEEE 60th Vehicular Technology Conference (VTC2004-Fall), vol. 2 Los Angeles, CA, USA, 26-29 Sept 2004, pp. 1129-1133

28. G Caire, RR Muller, R Knopp, Hard fairness versus proportional fairness in wireless communications: the single-cell case. Inform. Theory, IEEE Trans. 53(4), 1366-1385 (2007)

29. S Bai, W Zhang, Y Liu, Wang C, Max-min fair scheduling in, OFDMA-based multi-hop WiMAX mesh Networks, in Proceedings of IEEE International Conference on Communications (ICC) Kyoto, Japan, 5-9 June 2011, pp. 1-5 
30. P Phunchongharn, E Hossain, Distributed robust scheduling and power control for cognitive spatial-reuse TDMA networks. Selected Areas Commun. IEEE J. 30(10), 1934-1946 (2012)

31. S Peyman, H Simon, Robust transmit power control for cognitive radio. Proc. IEEE. 97(5), 915-939 (2009)

32. T Endeshaw, B Chalise, L Vandendorpe, Robust sum rate optimization for the downlink multiuser MIMO systems: worst-case design, in Proceedings of IEEE International Conference on Communications (ICC) Cape Town, South Africa, 23-27 May 2010, pp. 1-5

doi:10.1186/1687-1499-2014-69

Cite this article as: Liu et al:: Robust power allocation in two-tier heterogeneous networks. EURASIP Journal on Wireless Communications and Networking 2014 2014:69.

\section{Submit your manuscript to a SpringerOpen ${ }^{\circ}$ journal and benefit from:}

- Convenient online submission

Rigorous peer review

- Immediate publication on acceptance

- Open access: articles freely available online

- High visibility within the field

- Retaining the copyright to your article

Submit your next manuscript at $\boldsymbol{\triangleright}$ springeropen.com 